American Journal of Applied Sciences 5 (8): 1041-1046, 2008

ISSN 1546-9239

(C) 2008 Science Publications

\title{
Fracture and springback on Double Bulge Tube Hydro-Forming
}

\author{
F. Djavanroodi, M. Gheisary \\ Department of Mechanical Engineering, Iran University of Science and Technology, \\ Tehran, Iran
}

\begin{abstract}
This research aims to establish a basic understanding of Double Bulge Tube Hydro-Form processing of stainless steel deep drawn cups. The method is briefly reviewed by carrying out experimental tests and Finite element analysis. By measuring bulge height in both formed curves by Coordinate measuring machine (CMM) and thickness variation specimen by Ulterasonic thickness measurment device (UTM), it has been shown that maximum thinness occured where the bending is maximized. A finite element model is constructed to simulate the Double Bulge Tube Hydro Forming process and asses the influence of friction cofficient, tube Material properties and springback. It has been shown that material hardening coefficient had the most significant influence on formability characteristics during double bulge tube hydroforming. Also it is shown that springback has significant effect on tolerances of formed tube. Finally fracture strain was estimated by analytical method and compared with simulation results, also fracture location was predicted on Double Bulge Tube HydroForming (DBTHF) by simulating the process.
\end{abstract}

Keywords: Tube hydro forming, double bulge tube hydro forming, friction coefficient, strain hardening coefficient, springback, strain, fracture

\section{INTRODUCTION}

Tube Hydro-Forming is one of the hydro-forming applications and represents an excellent way of manufacturing complex automotive parts with a higher level of repeatability and lower tooling cost and provides means of structural component integration with package space efficiency ${ }^{[1]}$. Tube hydroformed parts have numerous advantages such as reduction in part weight, improved strength and stiffness and higher dimensional accuracy. This process has some drawbacks including slow cycle time, expensive equipment and lack of knowledge base for regarding the process and tool design ${ }^{[1]}$.

The hydroforming of double blanks is a process for forming hollow bodies. Hydraulic fluid is pumped between two blanks. The inner pressure forces the two blanks to move in separate directions towards the die ${ }^{[2]}$. A performing can also be done by conventional deep drawing, so it can be named as Double Bulge Tube Hydroforming. Schematic of Double blank tube hydroforming (parallel plate hydroforming) is shown in Fig. 1.

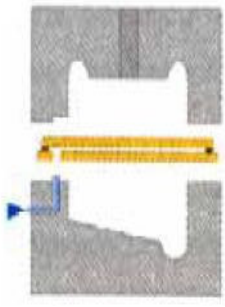

(a)

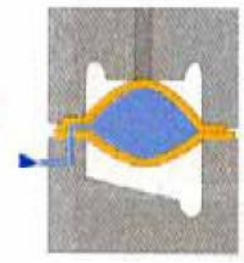

(b)

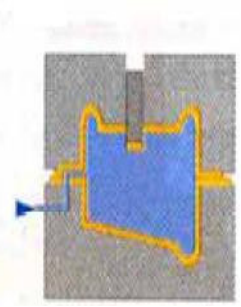

(c)
Fig 1: Schematic of the process sequence in double blank sheet hydroforming; a) close die, b) Forming, c) Calibration \& piercing $^{[5]}$

Lundkovist $^{[1]}$ in his thesis has presented a thorough literature survey of the tube hydroforming process and available simulation tools for the tube hydroforming process. Also he has investigated adaptive loading algorithms, the boundaries of the process window and speed up of the explicit finite element simulation.

Altan $^{[2]}$ has provided an overview on the advance in press (machines) and tools, tests for material and lubrication selection and warm hydroforming of magnesium and aluminum alloy sheets. He has shown that research and investigation of hydroforming of sheet

Corresponding Author: F. Djavanroodi, Department of Mechanical Engineering, Iran University of Science and Technology(IUST), Narmak, 16844, Tehran, Iran 
and tube have led to a) Improvement in accurate determination of material properties using tests that emulate the reality in production b) Development of better test methods to screen the lubricants c) Advances in press design that resulted in less expensive and compact presses with reduced cycle time d) Continuous improvement in tool design to increase the scope of applications of hydroforming and e) Development of virtual manufacturing tool through $\mathrm{FE}$ simulations to design the process and estimate the optimum process parameters.

Nielsen ${ }^{[3]}$, Harl $^{[4]}$ and Sigret ${ }^{[5]}$ studied different methods, advantages, Limitations and equipments in Tube Hydroforming Process.

Bruni $^{[6]}$ studied techniques in the evaluation of springback and residual stress in a TRIP800 steel cylindrical cup obtained by means of double sheet hydroforming. He has shown that high pressure, low corner radius and high friction (at the flange level), allowed the obtaining of high equivalent plastic strain on the top region of the samples, with the subsequent reduction in Springback.

Asnafi ${ }^{[7]}$ calculated optimized load path in force end THF by experimental, analytical and numerical methods. He used Stroke controlled method to analyze THF and has shown that the instability pressure and the instantaneous radius at the onset of instability, determined analytically, can be used as failure (fracture) criteria in free forming. Also Asnafi ${ }^{[8]}$ studied springback on double curved autobody panels. One can consider from his article that increasing the binder force, increasing the curvature, increasing the sheet thickness and/or decreasing the yield strength, reduces the springback.

In this paper, results of deep drawn cups 2nd forming sequence by DBTHF are presented. After measuring bulge Height in both formed curves by CMM and thickness variation of formed tube by Ultrasonic thickness measurement unit, a finite element model is constructed to simulate the DBTHF process. Also fracture strain is estimated and its location is predicted by simulation results.

\section{MATERIAL AND EXPERIMENTAL PROCEDURE}

The mechanical properties of materials that were examined are presented in Table 1. Deep drawn cups of $176.8 \mathrm{~mm}$ outer diameter, $0.68 \mathrm{~mm}$ wall thickness and $160 \mathrm{~mm}$ length made up of SS304 were tested and K and $\mathrm{n}$ values obtained from tensile tests according to
Table 1: Material properties

\begin{tabular}{ll}
\hline Stainless steel 304 & Mechanical Properties \\
\hline 82 & Hardness, Rockwell B \\
691.7 & Tensile Strength, Ultimate (MPa) \\
299.1 & Tensile Strength, Yield(Mpa) \\
$70 \%$ & Elongation at Break \\
207 & Modulus of Elasticity (Gpa) \\
818.48 & K(MPa) \\
0.2656 & $\mathrm{n}$ \\
\hline
\end{tabular}

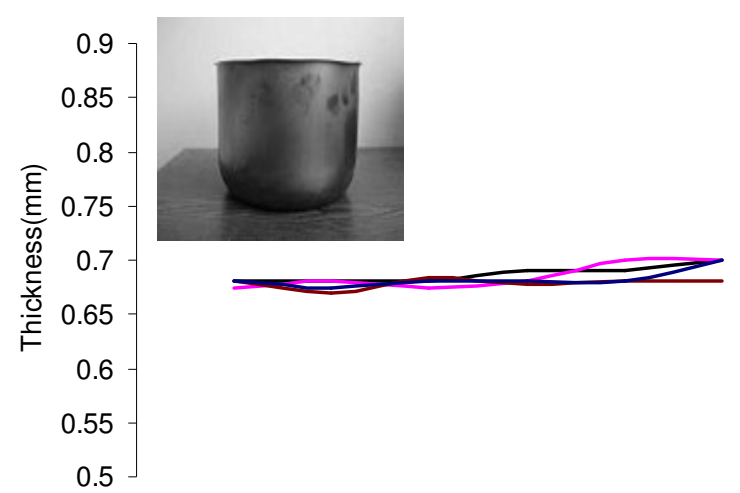

Fig. 2: Primary thickness variation (deep drawn cup)

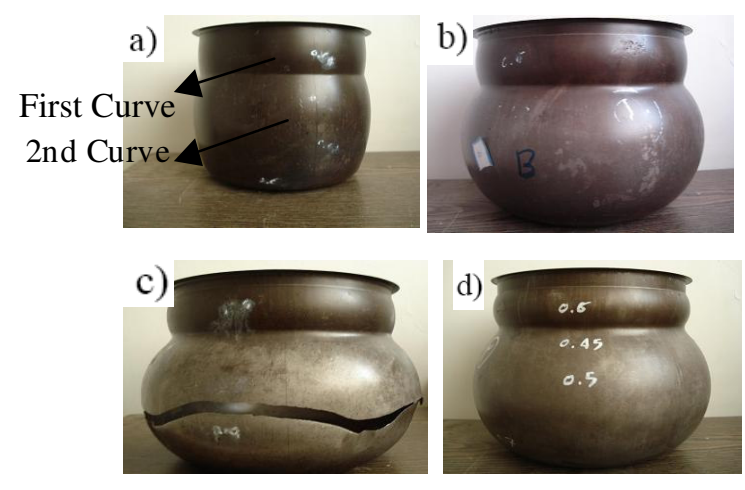

Fig. 3: Formed cups by DBTHF with different Internal Pressure (Bar); a) 55, b) 115, c) 135, d) Sample of burst cup

ASTM E8-M ${ }^{[9]}$. Using UTM unit, primary thickness variation of deep drawn cups which is used as blank were measured, it is evident that the blanks have uniform thickness Fig. 2. For DBTHF experiments, both ends of cup were fixed. After closing die parts, the tube was filled with oil and the air in the tube was evacuated. Number of formed specimen are shown in Fig. 3, these specimen are formed with different internal pressure. Thicknesses were measured in several points on the specimen with UTM unit. 


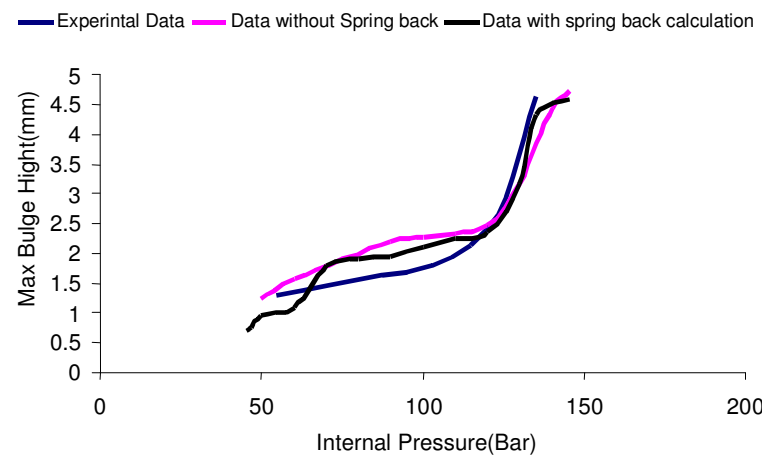

(a)

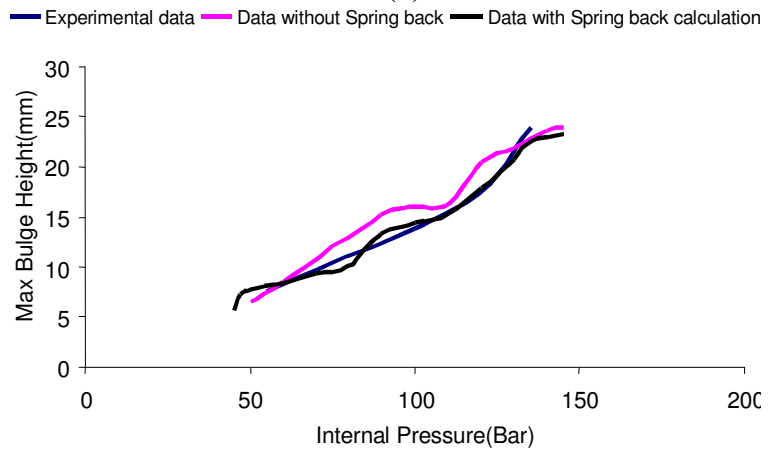

(b)

Fig. 4: Comparison of FE simulation results with experimental measurements of DBTHF of SS304 (a) first curve (b) second curve

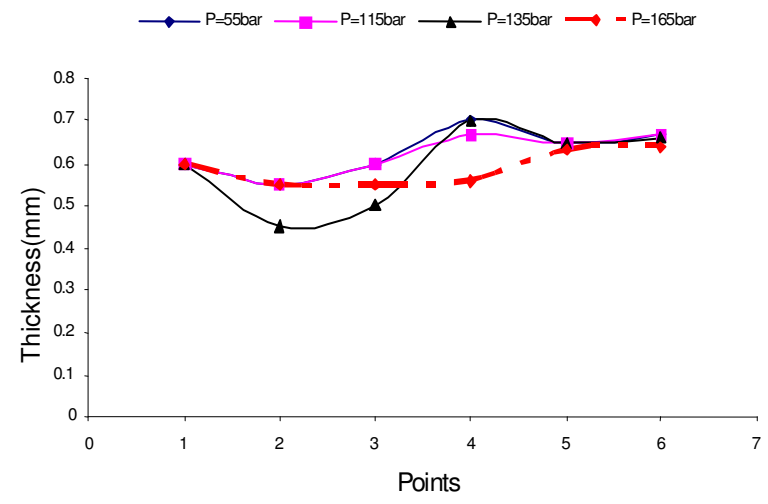

Fig. 5: Comparison of FE simulation results with experimental measurements of DBTHF

\section{RESULTS AND DISCUSSION}

FEM: Solid elements (deformable) were employed to model the Stainless steel cup and rigid element for die blocks, Power law constitutive model $\left(\sigma=K \varepsilon^{\mathrm{n}}\right)$ was utilized to model the material behavior. The values
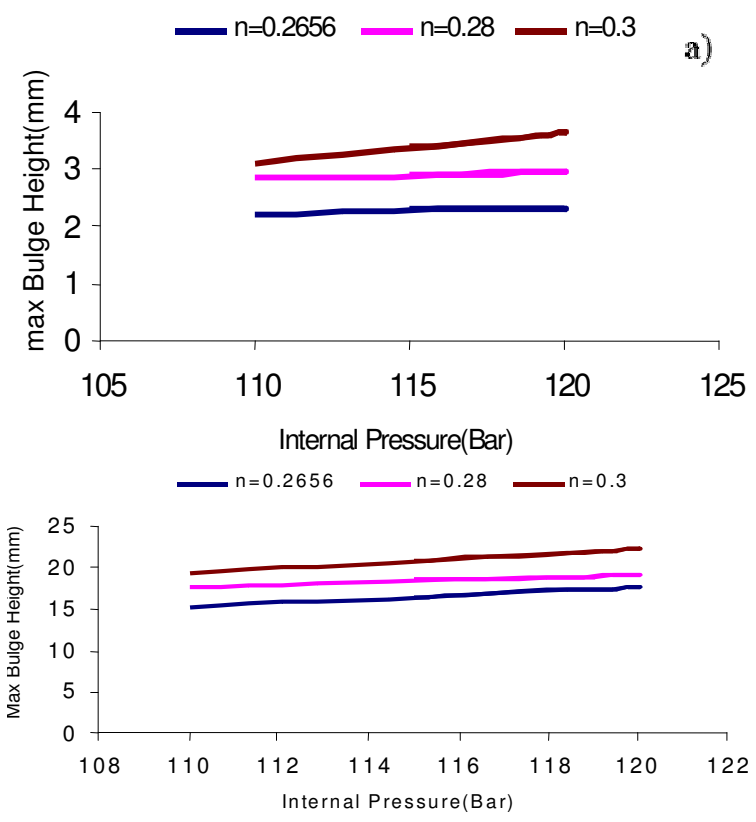

Fig. 6: Influence of hardening coefficient on formability of deep drawn cup; (a) first formed curve, (b) $2^{\text {nd }}$ formed curve

$\mathrm{K}=818.48$ Map and $\mathrm{n}=0.2656$ of the parent material were used in the calculations. A coulomb friction coefficient of $\mu=0.05^{[1,7,8]}$ was used for friction behavior between the contact areas of the tube and the die walls. Plots of bulge height versus internal pressure obtained from simulation for the two formed curved are shown in Fig. 4. For comparison purposes, the experimental measurements are also repeated in the same figure. Also Fig. 5 show comparison between FE simulation and experimental results in thickness variations at various height of the specimen (Point $1=140 \mathrm{~mm}$, point $2=120 \mathrm{~mm}$, point $3=100 \mathrm{~mm}$, point $4=160 \mathrm{~mm}$, point $5=50 \mathrm{~mm}$, and point $6=0 \mathrm{~mm}$ from bottom of cup respectively) for different pressures. It is clear that the FE simulations are in good agreement with the experimental results.

Strain hardening coefficient (n): To investigate the effect of hardening coefficient (n) on the formability of the deep drawn cups, the value of $n$ were varied between 0.26 to 0.3 and the corresponding bulge heights (both curves) with different internal pressures were compared. The resulting cup expansion is shown in Fig. 6. As illustrated, a larger hardening coefficient results in a higher expansion. Moreover, for a given increment in ' $n$ ' a greater increase in formability was seen at higher ' $n$ ' values. These results are in agreement 

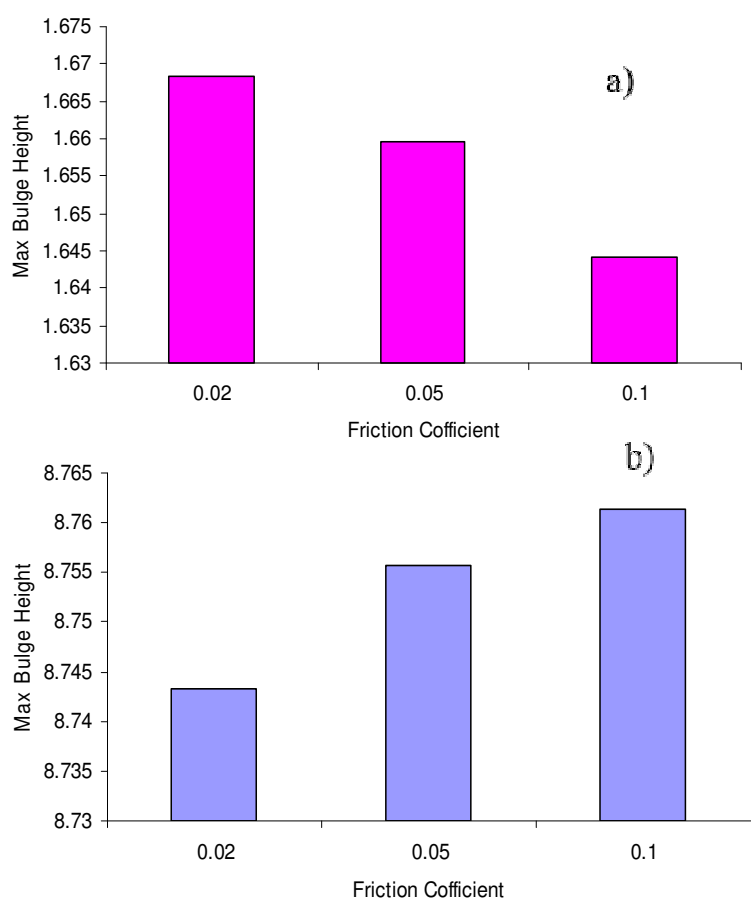

Fig. 7: Max outer diameter (bulge height) versus friction coefficient; a) first formed curve, b) $2^{\text {nd }}$ formed curve

with the known formability characteristics based on power law constitutive model for sheet metals. It can be concluded that material with higher hardening coefficient are always desirable so as to maximize the allowable expansion.

Influences of friction: Friction plays an important role in majority of forming operations. A low friction coefficient is often desirable for forming process. To study the effect of friction between the die and cup surfaces, different friction coefficients $(0.02,0.05$ and 0.1 ) were considered in the FE simulation. The resulting bulge heights plotted against the friction coefficient are shown in Fig. 7. As it can be seen, increasing of friction coefficient result in increasing bulge height in first formed curve, and decreasing maximum outer diameter in the 2 nd formed curve. In other words, because in the first sequences of forming process, 1st curve is formed earlier, more contact occurs between die walls and the cup in this region, so when forming region decreases in 1st curve, 2nd curve formability increases.

Springback on DBTHF: Better shape accuracy on formed tubes has been required and discussed for many
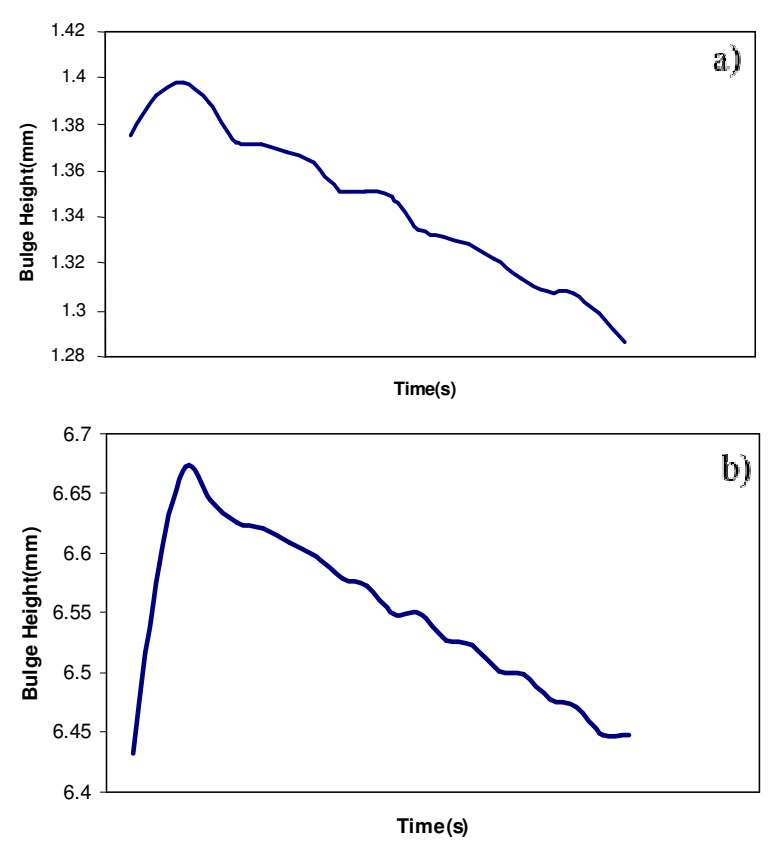

Fig. 8: Springback effect on Maximum outer diameter (bulge height), a) 1st formed curve, b) 2nd formed curve

years. It is, therefore, essential to clarify the factors governing tolerances on the formed tube. Spring back after forming processes, has a significant influence on the dimensions and tolerances of the formed tube. When the forming force is removed, higher yield stress, higher elastic strain and lower module of elasticity causes a higher springback value. For a specified metal with specified strain, springback value increases with higher width-thickness ratio. Moreover, heat treatment method, alloy components, die radius, bending angle and incline of real stress-strain diagram (contact module) show their effects on tolerances of final formed tube.

Figure 8 shows the changes of diameters of the specimen after removing the pressure, as it it is evident, when pressure increased, plastic zone is expanded and when pressure removed, maximum springback is appeared on the maximum bulge height. It should be noted that the length and radius of forming zone have significant influences on springback, more springback is recorded in the larger area, Fig. 9 shows the springback in the first and second formed curves. As illustrated, because 2nd curve form in primary sequences, plastic zone extracted and when internal pressure is removed, springback decrease, but first curve is yielded latter, therefore springback increase in this curve when forming force is removed. 

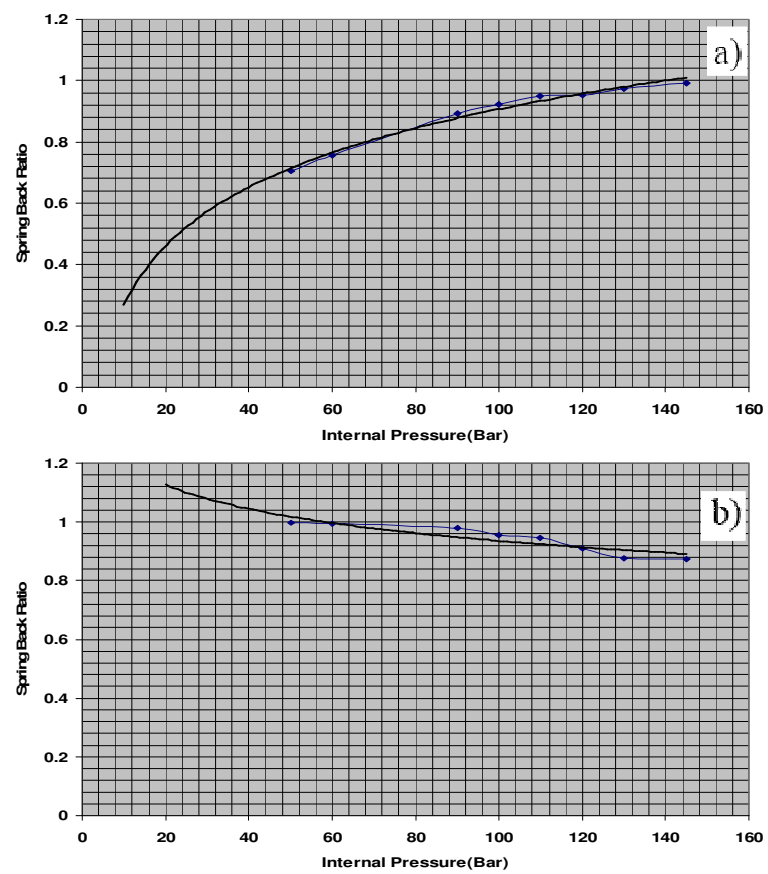

Fig. 9: Springback correction coefficient; (a) 1st formed curve, (b) 2nd formed curved

In Fig. 9 effect of springback correction has been removed form the FE analysis, and as it can be seen a better agreement has been reached between the experimental and numerical results.

Prediction of Fracture in DBTHF: The fracture strain in Tube Hydroforming (THF) is given by ${ }^{[7]}$ :

$$
\varepsilon_{1 f}=\frac{\left.(1+r) n-\sqrt{4 / 3} \operatorname{Ln}(1\lrcorner^{\mathrm{b})_{)_{-}}}\right)}{\left[\frac{4}{3}\left(1+\beta+\beta^{2}\right]^{1 / 2}\right.}
$$

Where $\varepsilon_{1 \mathrm{f}}$ is major strain at fracture, $\beta$ is strain ratio $\left(\varepsilon_{1} / \varepsilon_{2}\right)$ and $\mathrm{r}$ is plastic strain ratio.

If we use Von-Mises yielding criterion, the anisotropy is neglected and $r=1$, Equation (1) can be written as:

$$
\varepsilon_{1 \mathrm{f}}=\frac{2 \mathrm{n}-\sqrt{4 / 3} \operatorname{Ln}\left(1+\frac{\mathrm{t}_{0}}{\mathrm{~d}_{0}}\right)}{\left[\frac{4}{3}\left(1+\beta+\beta^{2}\right]^{1 / 2}\right)}
$$

Analytical and numerical solutions of bulge forming ${ }^{[7]}$ and calculating the strain ratio $\left(\varepsilon_{1} / \varepsilon_{2}\right)$ in different stages of forming, shows that $\beta=-0.5$ can be used to estimate fracture strain. As illustrated in Fig. 10, it is clear that the FE simulations are in good agreement with the experimental results. Bursting pressure in $\mathrm{FE}$ analysis is equal to 165 bar and in experiments this pressure is about 160 bar. The deviation in burst pressure is partly due to the calculation of fracture strain ( $\beta$ is for cylindrical shape strain ratio). Tensile stress fracture criterion is used and element deletion occurs when strain is equal to fracture strain. One can see that failure position in simulated shape and experimental formed tube, are in good agreement.
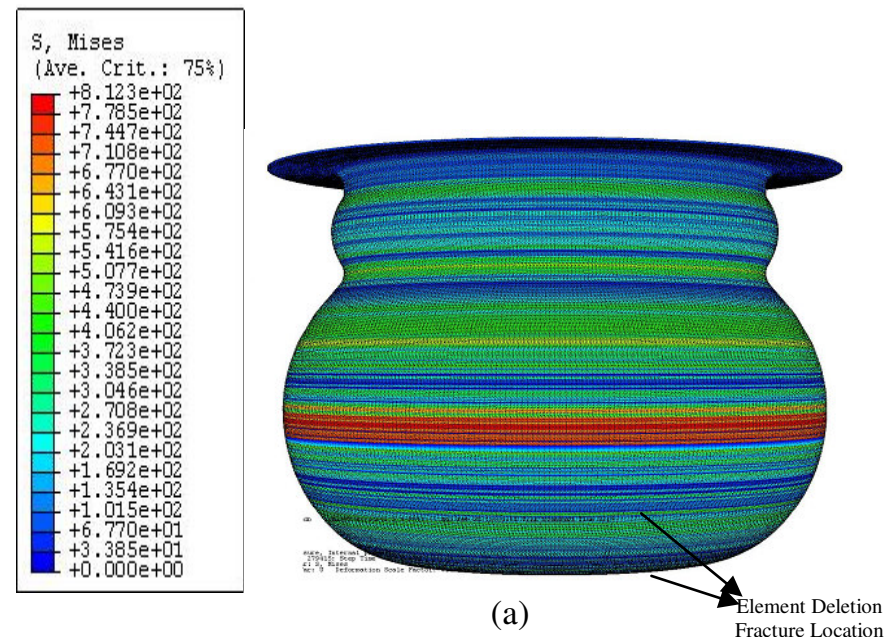

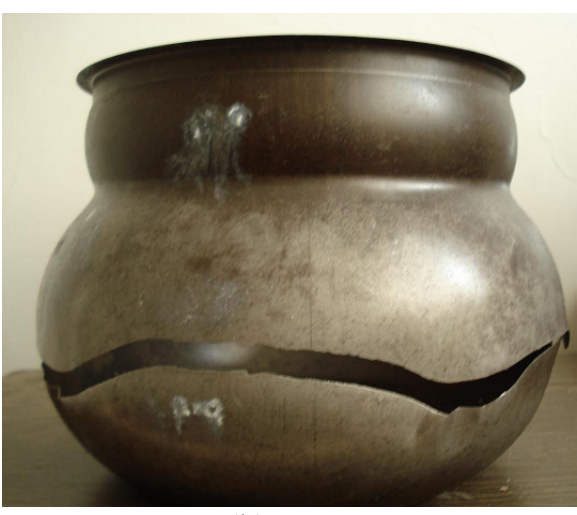

(b)

Fig.12: Bursting Location; (a) FE analysis, (b) Experimental 


\section{CONCLUSION}

Based on the above experimental and FE simulation results and subsequent discussions, the following conclusions are drawn:

- Strain hardening coefficient has the significant influence on formability of the tube, so that for forming materials with larger $\mathrm{n}$, low internal pressure is needed, but thickness variation in these materials is more than others with lower strain hardening coefficient

- When friction between die walls and tube increase, it causes renitent force on the contact surface, so max. outer diameter decreases and thickness variation increases

- For tight tolerances are on final formed tube, spring back should be controlled in process. With higher friction, larger elasticity module, bigger initial thickness, smaller Die radius and lower yielding stress, tighter tolerances can be obtained

- As shown, fracture estimation equation for other THF processes such as bulge forming, can be used to estimate the fracture on DBTHF. Also

- fracture zones in both simulation and experiment are in good agreement

- Similar to the other THF processes, using hydrostatic pressure in DBTHF, postpones the fracture and facilitate forming process

\section{REFERENCES}

1. Lundkovist, J., 2004. Numerical Simulation of Tube hydro forming, MSC Thesis.

2. Altan, T., 2002. Advance in Hydro forming for Manufacturing Automotive Part: Engineering Research center for Net shape manufacturing.

3. Nielsen, K.B., 2004. Hydro forming Highlights: sheet hydro forming and tube hydro forming: Journal of Material Processing Technology, 15: 165-177.

4. Hartl, Ch., 2005. Research and advances in fundamentals and industrial application of hydro forming: Journal of Material Processing Technology, 167: 383-392.

5. Sigeret, K., 2000. Recent Development in HydroForming Technol. J. Material Processing Technology, 48: 251-258.

6. Bruni, C., 2006. A study of techniques in the evaluation of springback and residual stress in hydroforming: International journal of advanced manufacturing technology, DOI 10.1007/s00170006-0539-x.

7. Asnafi, N., 2000. Theoretical and Experimental analysis of Stroke-controlled tube hydro forming: Journal of Material Science and engineering, A279(2000): 95-110.

8. Asnafi, N., 2001. On spring back of double-curved autobody panels: International J. Mechanical sci., 43: 5-37.

9. ASTM EM, 2001. American Standard for Testing Materials. 